\title{
Sexualität und Intimität bei depressiven Erkrankungen
}

\author{
Sex and intimacy in depression
}

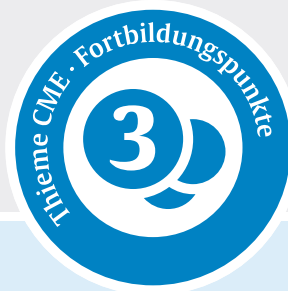

\author{
Andreas Ebert \\ LWL-Universitätsklinikum Bochum der Ruhr Universität \\ Bochum, Klinik für Psychiatrie, Psychotherapie und \\ Präventivmedizin
}

\section{ZUSAMMENFASSUNG}

Gegenstand und Ziel Sexuelle Bedürfnisse und Intimität beeinflussen die Lebensqualität von depressiven Patienten genauso wie die der Allgemeinbevölkerung. Trotz der hohen Prävalenz depressiver Störungen wird dieser Aspekt aus unterschiedlichen Gründen oft zu wenig beachtet. Besonders häufig sind Störungen der Libido bei depressiven Frauen wie Männern, es kann jedoch die ganze Bandbreite sexuellen Erlebens betroffen sein. Neben biologischen, psychologischen und partnerschaftlichen Ursachen für sexuelle Probleme ist insbesondere die pharmakologische Behandlung affektiver Störungen oft selbst Teil des Problems. Verschiedene medikamentöse und adjuvante Strategien können hier erfolgreich eingesetzt werden.

Schlussfolgerung und klinische Relevanz Nicht immer lassen sich sexuelle Nebenwirkungen, sexuelle Störungen oder Partnerschaftskonflikte vollständig verhindern. Dennoch sind ein offener und ehrlicher Kommunikationsstil und eine gründliche Prüfung von möglichen Vorgehensweisen, um dieses zusätzliche Leiden depressiver Patienten zu lindern, möglich. Nicht zuletzt ist eine weitere Erforschung des sexuellen Erlebens und der Intimität im Rahmen von Depressionen dringend erforderlich.

\section{Einleitung}

Ein erfülltes Sexualleben und das Verlangen nach Intimität stellen elementare menschliche Bedürfnisse dar. Unzufriedenheit in diesem Bereich reduziert die Lebenszufriedenheit auch in der Allgemeinbevölkerung deutlich [1]. Männer, die mit zunehmendem Lebensalter unter sexuellen Störungen leiden, empfinden nicht nur allgemein eine verringerte Lebensqualität, sondern leiden auch häufiger unter depressiven sowie Angstsymptomen [2]. Auch bei jüngeren Frauen sind depressive Symptome mit Störungen der Sexualfunktion assoziiert [3] und bei Frauen nach der Menopause besteht erwartungsgemäß ebenso ein Zusammenhang zwischen Sexualleben und der Lebensqualität [4].

Die Gründe und Ursachen für die teils erheblichen Einschränkungen im sexuellen Erleben bei depressiven Patienten sind vielfältig. Ganz offensichtlich haben die Hauptsymptome einer Depression wie Antriebsmangel oder Interessensverlust mit großer Wahrscheinlichkeit Folgen für das Intimleben der Patienten. Dennoch sind die genauen Zusammenhänge von biologischen, psychologischen, partnerschaftlichen und medikamentösen Faktoren sowie die Prävalenz von sexuellen Störungen bei klinischen depressiven Patienten überraschend wenig untersucht worden - trotz der Häufigkeit von Depressionen mit einer Lebenszeitprävalenz von etwa $20 \%$ [5]. Im klinischen Alltag wird dieses Thema trotz kontinuierlicher Präsenz sexueller Inhalte in den Medien ebenso leicht übersehen. Die möglichen Gründe dafür sind vielschichtig, dabei können Zeitmangel bei der Anamneseerhebung, eigene Hemmungen des Arztes oder des Therapeuten, dieses Thema anzuspre- chen oder gar das objektive Vorliegen dringlicherer Punkte (wie akute Suizidalität) eine Rolle spielen. Dieser Beitrag soll zum einen dazu beitragen, die Sensibilität für dieses Thema bei zukünftigen Patientenkontakten wieder zu erhöhen sowie zum anderen einen Überblick über das komplexe Zusammenspiel verschiedener Faktoren für die sexuelle Zufriedenheit und das Erleben von Intimität bei depressiven Menschen geben.

\section{Sexuelle und partnerschaftliche Probleme bei depressiven Erkrankungen}

Ungefähr die Hälfte der klinisch depressiven Patienten leidet bereits vor Einleitung einer medikamentösen Therapie unter Libidoverlust [6], wobei die Angaben hierzu in verschiedenen Untersuchungen deutlich schwanken [7]. In der Studie von Kennedy et al. [6] hatten fast die Hälfte der männlichen Teilnehmer zudem Schwierigkeiten, die Erektion aufrechtzuerhalten, Patientinnen litten fast genauso oft unter verminderter Lubrikation; merklich seltener traten bei beiden Geschlechtern Orgasmusstörungen (bzw. der Ejakulation) auf (15-22\%). Auch die Häufigkeit sexueller Fantasien ließ im Rahmen der depressiven Erkrankung nach. Allerdings sind Veränderungen des sexuellen Erlebens bei depressiven Patienten vielschichtig, so sind in der Literatur gar Fälle von Hypersexualität beschrieben worden [8].

Bereits die biologischen Veränderungen im Körper von depressiven Patienten machen Auswirkungen auf die Sexualität durch die Erkrankung wahrscheinlich. So steigen die Spiegel des Stresshormons Kortisol an [9]; die Hypothala- 
mus-Hypophysen-Nebennieren-Achse wird also aktiviert, dies ist möglicherweise besonders stark bei depressiven Patienten mit psychotischen Symptomen ausgeprägt [10]. Ob die Spiegel des Sexualhormons Testosteron hingegen, wie früher angenommen, bei depressiven Männern wirklich durchweg niedriger sind, ist inzwischen aufgrund einer heterogenen Studienlage unklar [11]. Allerdings wird die Gabe von Testosteron in besonderen Fällen zur Ergänzung einer antidepressiven Therapie mit ermutigenden Ergebnissen erprobt, sogar bei Patientinnen [12].

Die mit sexuellen Schwierigkeiten verbundenen erheblichen Belastungen, die depressive Erkrankungen für eine Partnerschaft mit sich bringen können, sind auch aus dem klinischen Alltag bekannt. Allerdings ist es mittels der häufigen Querschnittstudien kaum möglich, Ursachen und Folgen klar zu trennen; gerade da ein Wechselspiel von verschiedensten Faktoren vorliegt. So könnten sexuelle Störungen durch hormonelle Dysbalancen wie beispielsweise niedrige Oxytozinspiegel vor postpartalen Depressionen hervorgerufen werden und somit definierte biologische Ursachen haben [13], auch Einflüsse von außen könnten reaktiv zu einem „Oxytozinmangel“ führen. Auf der anderen Seite ist es ebenso gut vorstellbar, dass ein depressiver Partner als weniger attraktiv empfunden wird und daher das Sexualleben eines Paares erst durch diese Bewertung leidet. Menschen, die zu Depressionen neigen, ordnen sich hinsichtlich ihres eigenen Aussehens als unattraktiver ein, dies konnte auch in einer Studie belegt werden [14]. Interessanterweise zeigte dieselbe Studie aber auch, dass depressive Probanden von anderen nicht als unattraktiver eingestuft wurden, wobei natürlich ein „Rating“ in einer Studie nicht mit dem Empfinden des eigenen Partners gleichgesetzt werden kann. Ähnliche Schwierigkeiten mit der ursächlichen Zuschreibung treten auf, wenn Zusammenhänge zwischen dem Vorhandensein von Depressionen und dem Beziehungsstatus untersucht werden sollen: Zwar treten Depressionen häufiger nach Trennungen und Scheidungen auf, allerdings erhöhen depressive Erkrankungen eben auch das Risiko für Ehekrisen [15]. Eine kanadische Studie aus dem Jahr 2009 fand, dass verheiratete Männer weniger unter den Anzeichen von Depressionen litten als ledige Männer, allerdings konnte dies bei den verheirateten respektive ledigen Frauen nicht gezeigt werden [16]. Ob hier allerdings bei Männern eine feste Bindung quasi als „Antidepressivum“ wirkte oder andere Faktoren wie das Bildungsniveau der Frauen eine wesentliche Rolle spielten, konnte (nachvollziehbarerweise) nicht eindeutig geklärt werden.

Während auf der einen Seite (zumindest in den westlichen Ländern) Sexualität in der Gesellschaft omnipräsent zu sein scheint und Pornografie via Internet auch für Jugendliche jederzeit auf dem Smartphone verfügbar ist, so sind sexuelle Schwierigkeiten im Privaten längst nicht enttabuisiert. Auch häufiger Konsum von Pornografie muss dabei in vielen Fällen nicht per se als gesundheitsschädigend zu werten sein. Sogenannte „problematische“ Konsummuster könnten aber eher bei Menschen, die zu Depressionen neigen und ein niedriges Selbstwertgefühl aufweisen, vorkommen [17]. Wie zu erwarten, zeigt sich auch bei Patienten, die sich primär wegen Erektionsstörungen ärztliche Hilfe suchen, zu einem hohen Prozentsatz psychiatrische Auffälligkeiten wie depressive Symptome oder Ängste [18]. Bei jungen Männern scheint dieser Effekt noch ausgeprägter zu sein. Auch bei früh im Leben auftretenden sexuellen Störungen wie Erektionsstörungen sind die Zusammenhänge mit psychischen Symptomen komplex und es ist bereits hier meist ursächlich nicht zu trennen, ob nun die sexuellen Probleme z. B. einer depressiven Episode vorausgehen, oder in der Folge auftreten oder sich gegenseitig bedingen [19]. Hinzu kommt, dass sich das gesellschaftliche Klima dynamisch verändert, und damit die Wahrnehmung, Akzeptanz und subjektive Relevanz verschiedener Facetten des sexuellen Erlebens zu- oder abnehmen könnten - und damit auch deren Einfluss auf das psychische Wohlbefinden.

\section{Auswirkungen von Antidepressiva auf die Sexualität}

Mit der Einführung von Antipsychotika oder Antidepressiva konnten erstmals viele psychiatrische Patienten überhaupt suffizient behandelt werden. Ein Nebeneffekt des zunehmenden Einsatzes von ZNS-wirksamen Medikamenten in der Psychiatrie waren allerdings zunehmend auftretende sexuelle Nebenwirkungen, die nicht durch die ursprüngliche Krankheit, sondern erst durch die medikamentöse Behandlung ausgelöst wurden. Für depressive Patienten stellt sich die Lage dabei nur wenig besser als beispielsweise für Patienten mit Schizophrenie dar. Bereits die „älteren" trizyklischen Antidepressiva haben häufig sexuelle Nebenwirkungen, insbesondere Clomipramin [20]. Während etliche störende Nebenwirkungen der Trizyklika wie orthostatische Blutdruckabfälle, Sedierung oder Obstipation mit dem Markteintritt der selektiven Serotonin-Wiederaufnahmehemmer (SSRI) und später der selektiven Serotonin-Noradrenalin-Wiederaufnahmehemmer (SNRI) seltener wurden, so blieben die sexuellen Nebenwirkungen auch bei diesen neuen Substanzen erhalten oder sind gar ausgeprägter [21, 22]. Dies schränkt die Lebensqualität von vielen bezüglich der depressiven Grunderkrankung erfolgreich behandelten Patienten weiter ein. Nicht zuletzt führen die Nebenwirkungen häufig zu einer reduzierten Compliance von Seiten der Patienten, was zum Therapieabbruch führen kann (mit dem erheblichen Risiko eines Wiederauftretens der Depression) [23]. Wie schon bei den Trizyklika stehen bei den SSRI neben einer möglicherweise reduzierten Libido insbesondere Verzögerungen oder das Ausbleiben des Orgasmus bzw. der Ejakulation im Vordergrund. Die verzögernde Wirkung von SSRI auf die Ejakulation ist derart reproduzierbar, dass inzwischen mit Dapoxetin ein SSRI explizit zur Behandlung der vorzeitigen Ejakulation zugelassen ist und schon seit vielen Jahren auch 
andere SSRI (im „Off-Label-Use“) hierfür verordnet werden [24]. Viel Aufmerksamkeit hat dabei in den letzten Jahren das „Post-SSRI-Syndrom“ erfahren. Hierbei persistieren die unerwünschten sexuellen Wirkungen nach dem Beenden der Therapie mit SSRI oder treten damit verbunden erst auf [25]. Die Bedeutung und vor allem die Häufigkeit des Post-SSRI-Syndroms wird noch erforscht und ist teils umstritten. Jedoch führen nicht alle Antidepressiva in bedeutsamem Maße zu sexuellen Nebenwirkungen, sodass wie folgend besprochen, eine Reihe von wirksamen Strategien und Alternativen zur Verfügung stehen.

\section{Strategien zur Förderung der sexuellen Zufriedenheit bei Patienten mit depressiven Störungen}

Zuallererst steht die suffiziente Behandlung der Depression im Vordergrund. So kann und sollte eine leitliniengereichte Psychotherapie der depressiven Grunderkrankung erwogen werden. Mit dem Abklingen der Depression wäre auch eine Reduktion der sexuellen Symptome zu erwarten. Daneben können auch spezifische psychotherapeutische Techniken für sexuelle Störungen im Allgemeinen angewandt werden. Hierzu gibt es von klassischer Sexualtherapie über „Fertigkeiten“-Trainings bis zu kognitiver Verhaltenstherapie eine große Bandbreite an Verfahren. Die Studienlage zur Wirksamkeit ist allerdings uneinheitlich, sodass noch Forschungsbedarf besteht [26]. Eine erfolgreiche medikamentöse Therapie mit Antidepressiva kann analog zur Psychotherapie neben der positiven Wirkung auf gedrückte Stimmung oder Antriebsmangel prinzipiell auch sexuellen Symptome wie Libidoverlust reduzieren. Allerdings können wie beschrieben die sexuellen Nebenwirkungen von Antidepressiva hier zu neuen oder gar zusätzlichen Problemen führen [27]. Daher nimmt das Management der pharmakologischen Nebenwirkungen bei Depressionen einen besonderen Stellenwert ein. Selbstverständlich sollten somatische Ursachen wie Diabetes mellitus oder Substanzmissbrauch ausgeschlossen werden [21].

Bei einem Teil der Patienten können die sexuellen Nebenwirkungen nur vorrübergehender Natur sein, sodass zumindest initial ein bewusstes Abwarten eine mögliche Vorgehensweise sein kann. In anderen Fällen sind Dosisreduktionen oder andere Präparate sinnvoll [21]. Es gibt jedoch leider etliche Fälle, in denen eine medikamentöse Umstellung auf ein vom Nebenwirkungsprofil eigentlich geeigneteres Präparat nicht in Frage kommt. So führt zum Beispiel bei komorbider Zwangssymptomatik meist kein Weg an SSRI (oder Clomipramin) vorbei [28], die über den serotonergen Weg die typischen Nebenwirkungen wie verzögerte Ejakulation, reduzierte Orgasmusfähigkeit oder erniedrigte Libido haben können. Auch bei schweren oder therapieresistenten Depressionen müssen in der therapeutischen Gesamtabwägung oft sexuelle Nebenwirkungen bis zu einem gewissen Grad akzeptiert werden, um keine erneute Krise auszulösen (falls der Patient z. B. nur auf ein gewisses Präparat respondiert hat). In diesen Fällen kann neben nicht medikamentösen Optionen wie Psychotherapien oder supportive Maßnahmen die Verordnung von weiteren Medikamenten zur Behandlung der Nebenwirkungen erforderlich werden. Bei männlichen Patienten kann (nach Ausschluss körperlicher Ursachen) eine Verordnung von PDE-V-Hemmern wie Sildenafil in Frage kommen [29, 30]. Eine andere Möglichkeit ist die Ergänzung der Therapie mit SSRI durch eine Augmentation mit Bupropion, das als Dopamin-Noradrenalin-Wiederaufnahmehemmer einen anderen Wirkmechanismus besitzt und vielfach zur Reduktion sexueller Nebenwirkungen eingesetzt wird [31]. Hierbei ist es wichtig, dass Ärzte wie Psychotherapeuten das Schildern von sexuellen Nebenwirkungen ernst nehmen, zumal das Erwähnen der Nebenwirkungen aufgrund von Scham seitens des Patienten ohnehin unterbleiben könnte. Dabei gilt es sowohl, Compliance-Probleme zu vermeiden als auch zu verhindern, dass Patienten noch mehr das Gefühl haben, mit ihnen stimme etwas nicht (da sie z. B. die aufgetretenen sexuellen Schwierigkeiten nicht einer medikamentösen Wirkung zuordnen können).

Andererseits sollte aus Sorge vor potenziellen Nebenwirkungen eine antidepressive Therapie nicht ganz unterbleiben, insbesondere, wenn die Ausprägung der Symptomatik dies unbedingt erfordert. In einigen Fällen könnte eine verbesserte Stimmung, mehr Gelassenheit und weniger Grübeln infolge einer erfolgreichen Behandlung der Depression zur Folge haben, dass letztlich der Benefit für den Patienten auch bezüglich des Sexuallebens überwiegt, selbst wenn die eingesetzte Substanz von der pharmakologischen Wirkung eigentlich einen hemmenden Effekt auf die Sexualfunktion haben müsste.

Eine grundsätzliche Alternative bei der Psychopharmakotherapie können aber Antidepressiva sein, die weniger mit sexuellen Funktionsstörungen einhergehen wie das erwähnte Bupropion, Moclobemid, Agomelatin, (mit Einschränkungen) Mirtazapin oder andere [32-34]. Auch Trazodon [32, 35] und Tianeptin [36] scheinen seltener unerwünschte Wirkungen auf die Sexualfunktion zu haben. Nicht zuletzt kann in leichteren Fällen auch Johanniskraut-Trockenextrakt eine Alternative darstellen [37].

Neben dem Einsatz psychotherapeutischer Methoden und einer Optimierung der medikamentösen Therapie kommen unterstützend noch weitere supportive Maßnahmen in Betracht. Regelmäßige sportliche Betätigung und körperliche Aktivierung ist nicht nur aufgrund der allgemein bekannten gesundheitlichen Vorteile auch bei depressiven Patienten mit sexuellen Funktionsstörungen zu empfehlen. So scheint Sport zu einer verbesserten Sexualfunktion beizutragen und sexuelle Störungen zu reduzieren [ 38 , 39]. Neben physischer Fitness haben in den letzten Jahren vermehrt Meditation und achtsamkeitsbasierte Methoden an Beliebtheit und Verbreitung gewonnen. Aufgrund einer Vielzahl verschiedener Meditationsstile und wenig 
verfügbarer Daten wird Meditieren derzeit (noch) kaum spezifisch zur Förderung der sexuellen Gesundheit empfohlen. Allerdings gibt es für die Untergruppe der achtsamkeitsbasierten Verfahren eine deutlich bessere Datenlage: Eine 2019 erschienene Übersichtsarbeit fasst bereits 15 Publikationen zu achtsamkeitsbasierten Interventionen bei sexuellen Störungen zusammen [40]. Dies ist im Kontext des Themas Sexualität bei Depressionen vielversprechend, da die achtsamkeitsbasierte kognitive Therapie (mindfulness-based cognitive therapy, MBCT) bereits langjährig Anwendung in der Behandlung bzw. Prävention von Rückfällen depressiver Erkrankungen findet [41]. Für interessierte Patienten kann auch Yoga als ergänzende Maßnahme erwogen werden, sowohl aus theoretischen Erwägungen (Kombination von Achtsamkeitselementen und körperlicher Aktivierung) als auch aufgrund von ersten Untersuchungen, die einen Zusammenhang zwischen verbesserter Sexualfunktion und Yoga andeuten [42, 43].

\section{Diskussion}

Unter der Berücksichtigung der Häufigkeit depressiver Störungen [5] und der Folgen auf der wirtschaftlichen Makroebene [44] sowie der persönlichen Konsequenzen für die Betroffenen, zu denen eben auch gescheiterte Beziehungen zählen [15], mag es verwundern, wie wenig belastbare Untersuchungen es zu dem Thema Sexualität und Depression gibt. Hierfür kommen unterschiedliche Gründe wie gesellschaftliche und kulturelle Tabus, aber insbesondere auch die Schwierigkeit, die vielen Ursachen und Einflussfaktoren im Rahmen wissenschaftlicher Studien gründlich zu trennen und untersuchen zu können, in Betracht. Dennoch bzw. gerade deswegen ist hier noch viel Forschungsbedarf vorhanden, auch da sich die gesellschaftlichen Rahmenbedingungen in diesem Bereich schnell wandeln.

Allerdings gibt es gerade im Bereich der sexuellen Störungen im Zusammenhang mit Depressionen viele Ansatzpunkte, um den Patienten eine Hilfe anbieten zu können. Dies kann von der Inanspruchnahme einer Psychotherapie über supportive Maßnahmen zur Steigerung des sexuellen Wohlbefindens wie beispielsweise sportlicher Betätigung oder Achtsamkeitsübungen gehen. Aus ärztlicher Sicht beginnt dies jedoch in jedem Falle mit dem aktiven Nachfragen und Ansprechen bezüglich des sexuellen Erlebens. Idealerweise wird das Sexualleben des Patienten auch bei der initialen Auswahl eines Antidepressivums mit einbezogen, so können ggf. Umstellungen und Verzögerungen bei der Pharmakotherapie vermieden werden. Spätestens falls sexuelle Nebenwirkungen berichtet werden, sollte dann eine Abwägung zwischen diesen unerwünschten Wirkungen, der Lebensqualität des Patienten und einem möglichen Rückfallrisiko (auch durch möglicherweise verminderte Compliance [27]) erfolgen. Wichtig ist es hierbei jedoch, nicht unreflektiert eigene oder gesellschaftliche Normen auf den Patienten zu übertragen. So kann es für manche Patienten akzeptabel sein, zumindest zeitweise weniger sexuelles Verlangen zu empfinden und dafür eine stabile Stimmung zu erlangen oder wieder dem Beruf nachgehen zu können. Einem derartigen Patienten wäre mit einer „aufgedrängten“ Umstellung der Medikation und möglicher Verschlechterung kaum gedient. Manchmal bleibt auch zumindest vorrübergehend nur die Option, die Nebenwirkungen zu einem gewissen Grade zu akzeptieren, falls ein Patient ohne ein bestimmtes Präparat bzw. eine bestimmte Dosierung oder Kombination nicht stabilisiert werden könnte oder gar eine vitale Bedrohung durch Suizidgefährdung im Raum stünde. In den allermeisten Fällen wird sich jedoch zumindest auf längere Sicht eine medikamentöse Anpassung oder ein Ergänzen des Therapieregimes anbieten.

\section{FAZIT FÜR DIE PRAXIS}

Bei der initialen Behandlung eines akut depressiven Patienten steht im klinischen Alltag oft die Nachfrage nach dem Intimleben und eventuellen sexuellen Schwierigkeiten nicht im Mittelpunkt. Dies kann auch durchaus begründet sein. Im Akutfall (z. B. bei einer notfallmäßigen Vorstellung des Patienten oder während der stationären Behandlung einer suizidalen Krise) hat das Vermeiden eines akuten Schadens (wie eines Suizidversuchs) und die Wiederherstellung von allgemeinem Antrieb im ambulanten Rahmen sicher zunächst Vorrang. Allerdings ist insbesondere bei der Erörterung von aufrechterhaltenden Faktoren der Depression und der Planung der langfristigen Behandlung (z. B. Medikation) eine regelmäßige Evaluation des sexuellen Erlebens anzuraten. Letztlich sollte die sexuelle Zufriedenheit des Patienten trotz mancher Hemmungen bewusst in die Therapieplanung und die ärztliche Interaktion einbezogen werden, entsprechend der Bedeutung dieses intimen Aspektes für das Leben des depressiven Menschen und seiner Angehörigen.

Interessenkonflikt

\section{Erklärung zu finanziellen Interessen}

Forschungsförderung erhalten: ja; Honorar/geldwerten Vorteil für Referententätigkeit erhalten: nein; Bezahlter Berater/interner Schulungsreferent/Gehaltsempfänger: nein; Patent/Geschäftsanteile/Aktien (Autor/Partner, Ehepartner, Kinder) an Firma (Nicht-Sponsor der Veranstaltung): nein; Patent/Geschäftsanteile/Aktien (Autor/Partner, Ehepartner, Kinder) an Firma (Sponsor der Veranstaltung): nein.

Erklärung zu nicht finanziellen Interessen

Der Autor gibt an, dass kein Interessenkonflikt besteht. 


\section{Dr. med. Andreas Ebert}

LWL-Universitätsklinikum Bochum

Klinik für Psychiatrie, Psychotherapie und Präventivmedizin

Ruhr-Universität Bochum, Alexandrinenstr. 1

44791 Bochum, Deutschland

Tel. 0234/50770

andreas.ebert@rub.de

\section{Literatur}

[1] Buczak-Stec E, König HH, Hajek A. The link between sexual satisfaction and subjective well-being: a longitudinal perspective based on the German Ageing Survey. Qual Life Res 2019; 28: 3025-3035

[2] Lu Y, Fan S, Cui ], et al. The decline in sexual function, psychological disorders (anxiety and depression) and life satisfaction in older men: A cross-sectional study in a hospital-based population. Andrologia 2020; 52: e13559

[3] Wåhlin-Jacobsen S, Kristensen E, Pedersen AT, et al. Androgens and Psychosocial Factors Related to Sexual Dysfunctions in Premenopausal Women: 2016 ISSM Female Sexual Dysfunction Prize. J Sex Med 2017; 14: 366-379

[4] Thornton K, Chervenak J, Neal-Perry G. Menopause and Sexuality. Endocrinol Metab Clin North Am 2015; 44: 649-661

[5] Hasin DS, Sarvet AL, Meyers JL, et al. Epidemiology of Adult DSM-5 Major Depressive Disorder and Its Specifiers in the United States. JAMA Psychiatry 2018; 75: 336-346

[6] Kennedy SH, Dickens SE, Eisfeld BS, et al. Sexual dysfunction before antidepressant therapy in major depression. J Affect Disord 1999; 56: 201-208

[7] Williams K, Reynolds MF. Sexual dysfunction in major depression. CNS Spectr 2006; 11 (Suppl. 9): 19-23

[8] Raymond NC, Coleman E, Miner MH. Psychiatric comorbidity and compulsive/impulsive traits in compulsive sexual behavior. Compr Psychiatry 2003; 44: 370-380

[9] Jia Y, Liu L, Sheng C, et al. Increased Serum Levels of Cortisol and Inflammatory Cytokines in People With Depression. J Nerv Ment Dis 2019; 207: 271-276

[10] Keller ], Gomez R, Williams G, et al. HPA axis in major depression: cortisol, clinical symptomatology and genetic variation predict cognition. Mol Psychiatry 2017; 22: 527-536

[11] Walther A, Breidenstein J, Miller R. Association of Testosterone Treatment With Alleviation of Depressive Symptoms in Men: A Systematic Review and Meta-analysis. JAMA Psychiatry 2019; 76: 31-40.

[12] Miller KK, Perlis RH, Papakostas GI, et al. Low-dose transdermal testosterone augmentation therapy improves depression severity in women. CNS Spectr 2009; 14: 688-694

[13] Skrundz M, Bolten M, Nast I, et al. Plasma oxytocin concentration during pregnancy is associated with development of postpartum depression. Neuropsychopharmacology 2011; 36: 1886-1893

[14] Ehlinger PP, Blashill AJ. Self-perceived vs. actual physical attractiveness: Associations with depression as a function of sexual orientation. J Affect Disord 2016; 189: 70-76

[15] Bulloch AG, Williams JV, Lavorato DH, et al. The relationship between major depression and marital disruption is bidirectional. Depress Anxiety 2009; 26: 1172-1177
[16] St John PD, Montgomery PR. Marital status, partner satisfaction, and depressive symptoms in older men and women. Can J Psychiatry 2009; 54: 487-492

[17] Bőthe B, Tóth-Király I, Potenza MN, et al. High-Frequency Pornography Use May Not Always Be Problematic. J Sex Med 2020; 17: 793-811

[18] Yang Y, Song Y, Lu Y, et al. Associations between erectile dysfunction and psychological disorders (depression and anxiety): A cross-sectional study in a Chinese population. Andrologia 2019; 51: e13395. doi:10.1111/and.13395

[19] Jern P, Gunst A, Sandnabba K, et al. Are early and current erectile problems associated with anxiety and depression in young men? A retrospective self-report study. J Sex Marital Ther 2012; 38: 349-364

[20] Segraves RT, Balon R. Antidepressant-induced sexual dysfunction in men. Pharmacol Biochem Beha 2014; 121: 132-137

[21] Atmaca M. Selective Serotonin Reuptake Inhibitor-Induced Sexual Dysfunction: Current Management Perspectives. Neuropsychiatr Dis Treat 2020; 16: 1043-1050

[22] Rothmore J. Antidepressant-induced sexual dysfunction. Med J Aust 2020; 212: 329-334

[23] Taylor M], Rudkin L, Bullemor-Day P, et al. Strategies for managing sexual dysfunction induced by antidepressant medication. Cochrane Database Syst Rev 2013. doi:10.1002/14651858.CD003382.pub3

[24] Cooper K, Martyn-St James M, Kaltenthaler E, et al. Interventions to treat premature ejaculation: a systematic review short report. Health Technol Assess 2015; 19: 1-180. doi:10.3310/hta19210

[25] Reisman Y. Sexual Consequences of Post-SSRI Syndrome. Sex Med Rev 2017; 5: 429-433

[26] Frühauf S, Gerger H, Schmidt HM, et al. Efficacy of psychological interventions for sexual dysfunction: a systematic review and meta-analysis. Arch Sex Behav 2013; 42: 915-933

[27] Clayton AH, El Haddad S, Iluonakhamhe JP, et al. Sexual dysfunction associated with major depressive disorder and antidepressant treatment. Expert Opin Drug Saf 2014; 13: 1361-1374

[28] Del Casale A, Sorice S, Padovano A, et al. Psychopharmacological Treatment of Obsessive-Compulsive Disorder (OCD). Curr Neuropharmacol 2019; 17: 710-736

[29] Fava M, Nurnberg HG, Seidman SN, et al. Efficacy and safety of sildenafil in men with serotonergic antidepressant-associated erectile dysfunction: results from a randomized, double-blind, placebo-controlled trial. J Clin Psychiatry 2006; 67: $240-246$

[30] Nurnberg HG, Hensley PL, Gelenberg AJ, et al. Treatment of antidepressant-associated sexual dysfunction with sildenafil: a randomized controlled trial. JAMA 2003; 289: 56-64

[31] Zisook S, Rush AJ, Haight BR, et al. Use of bupropion in combination with serotonin reuptake inhibitors. Biol Psychiatry 2006; 59: 203-210

[32] Chokka PR, Hankey JR. Assessment and management of sexual dysfunction in the context of depression. Ther Adv Psychopharmacol 2018; 8: 13-23

[33] Bonnet U. Moclobemide: therapeutic use and clinical studies. CNS Drug Rev 2003; 9: 97-140

[34] Watanabe N, Omori IM, Nakagawa A, et al. Mirtazapine versus other antidepressive agents for depression. Cochrane Database Syst Rev 2011. doi10.1002/14651858.CD006528. pub2 
[35] Fagiolini A, Comandini A, Catena Dell'Osso M, et al. Rediscovering trazodone for the treatment of major depressive disorder. CNS Drugs 2012; 26: 1033-1049

[36] Atmaca M, Kuloglu M, Tezcan E. Switching to tianeptine in patients with antidepressant-induced sexual dysfunction. Hum Psychopharmacol 2003; 18: 277-280

[37] Kasper S, Gastpar M, Möller HJ, et al. Better tolerability of St. John's wort extract WS 5570 compared to treatment with SSRIs: a reanalysis of data from controlled clinical trials in acute major depression. Int Clin Psychopharmacol 2010; 25 : 204-213

[38] Fergus KB, Gaither TW, Baradaran N, et al. Exercise Improves Self-Reported Sexual Function Among Physically Active Adults. J Sex Med 2019; 16: 1236-1245

[39] Stanton AM, Handy AB, Meston CM. The Effects of Exercise on Sexual Function in Women. Sex Med Rev 2018; 6: $548-557$

[40] Jaderek I, Lew-Starowicz M. A Systematic Review on Mindfulness Meditation-Based Interventions for Sexual Dysfunctions. J Sex Med 2019; 16: 1581-1596

[41] Teasdale JD, Segal ZV, Williams JM, et al. Prevention of relapse/recurrence in major depression by mindfulness-based cognitive therapy. J Consult Clin Psychol 2000; 68: 615-623
[42] Dhikav V, Karmarkar G, Verma M, et al. Yoga in male sexual functioning: a noncompararive pilot study. J Sex Med 2010; 7: 3460-3466

[43] Dhikav V, Karmarkar G, Gupta R, et al. Yoga in female sexual functions. J Sex Med 2010; 7: 964-970

[44] Greenberg PE, Fournier AA, Sisitsky T, et al. The economic burden of adults with major depressive disorder in the United States (2005 and 2010). J Clin Psychiatry 2015; 76: 155-162

Bibliografie

Nervenheilkunde 2021; 40: 981-986

DOI 10.1055/a-1637-9009

ISSN 0722-1541

(C) 2021. Thieme. All rights reserved.

Georg Thieme Verlag KG, Rüdigerstraße 14,

70469 Stuttgart, Germany 


\section{Punkte sammeln auf CM/F.thieme.de}

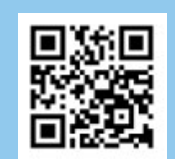

Diese Fortbildungseinheit ist in der Regel 12 Monate online für die Teilnahme verfügbar. Unter https://eref.thieme.de/CXIIRQN oder über den QR-Code kommen Sie direkt zur Startseite des Wissenstests und zum Artikel. Sie finden dort auch den genauen Einsendeschluss.

Sollten Sie Fragen zur Online-Teilnahme haben, finden Sie unter https://cme.thieme.de/hilfe eine ausführliche Anleitung.

Wir wünschen viel Erfolg beim Beantworten der Fragen!

VNR 2760512021160211227

\section{Frage 1}

Wahrscheinliche Ursachen für sexuelle Störungen im Zusammenhang mit Depressionen sind nicht:
A Hormonelle Dysbalancen
B Antidepressiva
C Yoga- und Achtsamkeitstrainingsprogramme
D Antipsychotika
E Probleme in der Partnerschaft

\section{Frage 2}

Welche Aussage ist richtig?

A Die Verordnung von SSRI führt bei ca. 70\% der Männer zu vorzeitiger Ejakulation.

B Beim Auftreten von sexuellen Nebenwirkungen im Zusammenhang mit Antidepressiva kann der Einsatz von Bupropion geprüft werden.

C Bei sexuellen Störungen, die bei depressiven Patienten vorliegen, ist es in den meisten Fällen möglich, eine eindeutige Ursache (wie hormonelle Probleme) festzustellen.

D Um die Scham von Patienten nicht noch weiter zu verstärken, sollte das Thema Sexualität nie bei den ersten Terminen angesprochen werden.

E Bei jungen männlichen Patienten sind sexuelle Probleme im Kontext psychischer Erkrankungen sehr selten.

\section{Frage 3}

Welche Aussage zu Medikamenten ist richtig?

A Im Gegensatz zu Antidepressiva treten bei klassischen Antipsychotika sexuelle Nebenwirkungen nur sehr selten auf.

B Dapoxetin wird häufig bei Zwangsstörungen eingesetzt.

C Sildenafil ist keine Therapieoption bei sexuellen Störungen, die durch Medikamente induziert sind.

D Wenn die antidepressive Wirkung einsetzt, treten sexuelle Nebenwirkungen kaum mehr auf.

E Moclobemid führt seltener zu sexuellen Nebenwirkungen als Serotonin-Wiederaufnahmehemmer.

\section{Frage 4}

Welche Aussage ist bezüglich des Vorgehens bei sexuellen Problemen im Kontext psychischer Störungen nicht zutreffend?

A Eine Umstellung auf andere Präparate kann sinnvoll sein.

B Neben medikamentösen Maßnahmen sollte auch eine Psychotherapie erwogen werden.

C Neuere Metaanalysen schließen einen möglichen positiven Einfluss von sportlicher Betätigung auf die sexuelle Funktionsfähigkeit aus, falls der jeweilige Patient komorbid an Depressionen leidet.

D Bezüglich der Wirksamkeit und vor allem der Auswahl der entsprechenden psychotherapeutischen Verfahren zur Behandlung sexueller Probleme besteht noch großer Forschungsbedarf.

E Mögliche körperliche Ursachen wie Diabetes mellitus oder arterielle Hypertonie sollten bei Diagnostik und Therapie ebenfalls erfragt und ggf. therapiert werden.

\section{Frage 5}

Welche dieser 3 Substanzen werden (verhältnismäßig) weniger mit sexuellen Nebenwirkungen wie Libidoverlust oder Orgasmusstörungen in Verbindung gebracht?
A Tianeptin, Mirtazapin, Trazodon
B Escitalopram, Paroxetin, Sildenafil
C Duloxetin, Clomipramin, Benperidol
D Fluvoxamin, Fluoxetin, Citalopram
E Dapoxetin, Venlafaxin, Milnacipran 
Punkte sammeln auf CME.thieme.de

Fortsetzung ...

\section{Frage 6}

Welche Aussage trifft am ehesten zu?

A Idealerweise wird das Thema Sexualität bereits frühzeitig angesprochen, auch trotz eigener Hemmungen des Arztes oder Therapeuten.

B Einschränkungen des Sexuallebens beeinträchtigen die Lebensqualität von Männern im Gegensatz zu der von Frauen erheblich.

C Sexuelle Probleme haben bei depressiven Patienten nur selten Auswirkungen auf die Partnerschaft, da die Partner der Patienten wissen, dass Depressionen und Sexualität eng verknüpft sind.

D Supportive Maßnahmen zur Steigerung des sexuellen Wohlbefindens sind während einer depressiven Episode kontraindiziert, da der Patient geschont werden sollte.

E Die sexuelle Zufriedenheit des Patienten sollte frühestens nach Remission der depressiven Symptomatik erfragt werden, da die Patienten dies während einer depressiven Episode ohnehin nicht einschätzen können.

\section{Frage 7}

Welche der folgenden Aussage trifft am wenigsten zu?

A Auch unabhängig von der Gabe von Psychopharmaka wie Antidepressiva leiden viele Patienten mit Depressionen an Symptomen wie Libidoverlust.

B Im Rahmen von Depressionen scheinen auch sexuelle Fantasien seltener zu werden.

C Der Testosteronwert im Serum ist bei Männern, die an Depressionen leiden, praktisch immer verringert.

D Je nach Studie liegt die Lebenszeitprävalenz von Depressionen bei etwa $20 \%$.

E Das Neuropeptid Oxytozin wurde mit Depressionen und sexuellen Störungen in Verbindung gebracht.

\section{Frage 8}

Diese Aussage zu Antidepressiva trifft am ehesten zu:

A Mit der Markteinführung von SSRI und später SNRI wurden zwar die sexuellen Nebenwirkungen seltener, dafür traten aber Nebenwirkungen wie Obstipation, orthostatische Hypotonie oder Sedierung im Gegensatz zu den trizyklischen Antidepressiva häufiger auf.

B SSRI können die Ejakulation verzögern, daher werden sie auch in der Therapie der vorzeitigen Ejakulation eingesetzt.

C Das „Post-SSRI-Syndrom“ ist bezüglich Relevanz, Häufigkeit und Konsequenzen bereits ausreichend erforscht, da es bereits seit der Einführung von Fluoxetin routinemäßig erfragt wurde.

D Falls sexuelle Symptome berichtet werden, ist von einer Behandlung mit Antidepressiva abzusehen.

E Agomelatin kann keine Therapieoption sein, wenn sexuelle Probleme unter der Behandlung mit SSRI auftreten.

\section{Frage 9}

Wenn sexuelle Nebenwirkungen bei einer antidepressiven Therapie auftreten, so ...

A sollte nie eine Dosisreduktion des bereits verordneten Medikaments versucht warden.

B kann ein Abwarten unter Beobachtung jedoch nicht in Frage kommen, da Nebenwirkungen praktisch nie zeitlich begrenzt sind.

C kann es bei sehr schwerwiegenden Fällen oder bestimmten Störungsbildern leider zeitweise notwendig sein, bestimmte Nebenwirkungen zu tolerieren, wenn es keine vielversprechende Therapieoption gibt oder die Schwere der Erkrankung eine Umstellung gerade nicht zulässt.

D darf dennoch eine Psychotherapie nicht als Alternative angesehen werden, da Depressionen und Panikstörungen in jedem Fall eine medikamentöse Behandlung erfordern.

E ist die Wirkung von achtsamkeitsbasierten Verfahren bei sexuellen Nebenwirkungen so gut belegt, dass eine Umstellung auf andere Substanzen nur selten erwogen werden muss.

\section{Frage 10}

Was trifft bezüglich sexueller Nebenwirkungen von Psychopharmaka zu?

A Ein „Post-SSRI-Syndrom“ kennzeichnet ein erneutes Auftreten von depressiven Kernsymptomen wie Antriebsmangel und Interesseverlust bei gleichzeitig wieder normaler Sexualfunktion.

B Clomipramin als trizyklisches Antidepressivum ist insbesondere aufgrund der nur seltenen Beeinträchtigung der Sexualfunktion im Gegensatz zu SSRI das Mittel der ersten Wahl bei Zwangsstörungen.

C Bei Panikstörungen verursachen Antidepressiva aufgrund des sogenannten „Teufelskreises der Angst“ wesentlich seltener sexuelle Nebenwirkungen.

D SNRI sind im Gegensatz zu SSRI wesentlich seltener mit sexuellen Nebenwirkungen assoziiert.

E Sexuelle Nebenwirkungen von Psychopharmaka können zu einer verringerten Compliance von Seiten des Patienten führen. 